\title{
An assessment of quality of life for early phase after adjuvant radiotherapy in breast cancer survivors: a Korean multicenter survey (KROG 14-09)
}

Chai Hong Rim', Sung-Ja Ahn², Jin Hee Kim³ ${ }^{3}$ Won Sup Yoon ${ }^{4 *}$, Mison Chun ${ }^{5}$, Dae Sik Yang ${ }^{6}$, Jong-Hoon Lee Kyubo Kim ${ }^{8,9}$, Moonkyoo Kong ${ }^{10}$, Suzy Kim" ${ }^{11}$, Juree Kim ${ }^{12}$, Kyung Ran Park ${ }^{9}$, Young-Joo Shin ${ }^{13}$, Sun Young Ma ${ }^{14}$, Bae-Kwon Jeong ${ }^{15}$, Su Ssan Kim ${ }^{16}$, Yong Bae Kim ${ }^{1}$, Dong Soo Lee ${ }^{17}$ and Jaehyung Cha ${ }^{18}$

\begin{abstract}
Backgrounds: Quality of life (QoL) has become a major concern as the survival time of breast cancer increases. We investigated the changes in QoL through comprehensive categorical analysis, for the first three years after breast cancer treatment including radiotherapy.

Methods: A total of 1156 patients were enrolled from 17 institutions. All survivors were grouped according to a surveillance period of 9-15 months (first year), 21-27 months (second year), and 33-39 months (third year) from the end of radiotherapy. The 5-dimensional questionnaire by the EuroQol group (EQ-5D) and the EORTC Quality of Life Questionnaire; breast cancer specific module (QLQ-BR23) were checked by self-administrated method.

Results: First, second and third year groups comprised 51.0, 28.9, and 21.0\%. In EQ-5D-3 L (3-Likert scale) analysis, pain/discomfort and anxiety/depression categories showed lower QoL. In multivariate analyses of EQ-5D-VAS (visual-analogue scale), categories of pain/discomfort and self-care were improved with time; axillary dissection was a significant clinical factor deteriorates pain/discomfort, self-care and usual activities. In QLQ-BR23 analysis, the lowest scored category was sexual activity, followed by sexual enjoyment, future perspective, and hair loss, and the best scored category was breast symptoms. In multivariate analyses, arm symptoms, breast symptoms and body image were improved with time.
\end{abstract}

Conclusions: Categories of pain/discomfort and self-care in EQ-5D-VAS, arm/breast symptoms and body image in QLQ-BR23 were improved, while categories of anxiety/depression and future perspective BR23 were not, suggesting necessity of psychosocial support. This research provides comprehensive information on the categorical aspects of QoL and changes during early follow-up after breast cancer treatment.

Keywords: Breast neoplasm, Quality of life, Radiation therapy, EQ-5D, EORTC-QLQ-BR23

\footnotetext{
*Correspondence: irionyws@korea.ac.kr

${ }^{4}$ Department of Radiation Oncology, Ansan Hospital, Korea University, 123

Jeokgeum-ro, Danwon-gu, Ansan, Gyeonggi-do 15355, Republic of Korea

Full list of author information is available at the end of the article
} 


\section{Backgrounds}

Breast cancer is the most frequently diagnosed cancer among females worldwide, and accounts for $25 \%$ of all cancer cases [1]. After a continuous increase during the 1980 's to 1990's, the incidence rates of Western countries in early 2000's declined or were stable, likely because of the reduced use of female hormones or plateaus in participation of mammographic screening. Death rates have been declining due to early detection or improved treatment [2]. In South Korea, similar tendencies of incidence and mortality were observed. The incidence has been continuously rising by $5.6 \%$ per year from 1999 to 2013 , and the 5-year survival rate was $91.5 \%$ between 2009 and 2013 comparing the 5-year survival rate of 77.9\% between 1993 and 1995 [3].

Since there have been significant increases in the survival of breast cancer patients, there are emerging issues concerning quality of life (QoL) after surgery and adjuvant treatment [4]. Besides the progress of surgical methods, chemotherapy and hormone therapy, adjuvant radiotherapy plays an important role to improve overall survival. Recent long-term follow-up outcomes reconfirmed the survival gains of adjuvant radiotherapy $[5,6]$.

Previous studies revealed that breast cancer survivors continue to experience adverse effect of cancer itself and treatment years after the treatment finishes, including fatigue, pain, fear of recurrence, depressive symptoms, and sexual discomfort. All those symptoms become a hindrance to the recovery of patients' daily activities or self-management $[7,8]$.

However, most data about QoL of breast cancer patients derived from cross-sectional studies, which are not able to indicate the course of QoL over time. Some longitudinal studies about QoL of breast cancer patients might have the drawbacks of a small study population with a limited statistical power or outdated version to present recent therapeutic principle [7, 8]. Additionally, there are very few studies that are based on the general QoL after adjuvant radiotherapy.

As the concern regarding the QoL of cancer survivors is rising, we designed a nationwide multi-institutional study to examine the QoL of breast cancer survivors, in the critical early phase up to 3 years after a completion of adjuvant radiotherapy.

\section{Methods}

\section{Study population recruitment}

The population of our study consisted of patients diagnosed with breast cancer, treated with definite surgery followed by adjuvant radiotherapy, regardless of any other adjuvant. We performed a questionnaire survey to patients who visited the outpatient clinic and corresponded to the periods of 3 months before and after 1, 2 , and 3 years after completion of radiotherapy. For example, the population in the 1st year group visited from 9 to 15 months after completion of radiotherapy. The included patients in our study were who; 1) successfully completed planned radiotherapy; 2) were aged between 20 and 70 at the initiation of radiotherapy; 3) were able to communicate in the Korean language and 4) agreed to answer our questionnaire. Exclusion criteria were those who; 1) had any locoregional recurrence or metastasis during or after radiotherapy; 2) had a history of any malignancy except non-melanoma skin cancer; 3) had a disability below Eastern Cooperative Oncology Group performance scale 2 due to physical problem other than breast cancer; and 4) is male.

The population size of our study was estimated using our country and United States cancer statistics. In the year 2011 in South Korea, the newly diagnosed breast cancer patients totaled 15,356, and 2063 people died due to breast cancer. Based on the above values, we assumed that about 13,000 breast cancer patients survived each year [9]. According to the Surveillance, Epidemiology, and End Results Program data of United States, 51\% patients with early breast cancer (stage I or II) and $44 \%$ of advanced breast cancer (stage III or IV) received adjuvant radiotherapy [10]. Considering this, we expect that about $50 \%$ of breast cancer patients would receive adjuvant radiotherapy in South Korea, and we presumed that the number was about 6500 patients each year. Regarding that our exclusion criteria may exclude about 500 patients in each year group, we can estimate that the parent population would be about 6000 patients in a year. With this estimation and the following formula, we calculated the size of sample for our study.

$$
n=z_{a / 2}^{2}{ }^{*} 0.5^{2} /\left\{(\text { Sampling error })^{2}+z_{a / 2}^{2}{ }^{*} 0.5^{2} / N\right\}
$$

(Confidence level 90\% $\mathrm{z}_{\mathrm{a} / 2}=1.65$, Confidence level $95 \% \mathrm{z}_{\mathrm{a} / 2}=1.96$, Confidence level $99 \% \mathrm{z}_{\mathrm{a} / 2}=2.54$, $\mathrm{N}=$ parent population).

As we used a confidence level of $95 \%$ and sampling error of $+/-5 \%$, we calculated that 384 patients was the sample size needed per year. Consequently, the ideal size of sample for 3 years is 1152. Hence, our goal was to perform our survey to about 1100 patients. However, due to the characteristic of clinical follow-up that the rate of regular checkup is declining as time passes, we applied sample errors and set the sample sizes as followed; 1) 317-474 survivors at the 1 st year with $+/-4.5-5.5 \%$ sample error; 2) 227-317 survivors at the 2nd year with $+/-5.5-6.5 \%$ sample error; and 3) 170-227 survivors at the 3 rd year with $+/-6.5-7.5 \%$ sample error.

\section{QoL measurement}

The QoL of study participants was assessed using the 5dimensional questionnaire by EuroQol group (EQ-5D) 
to evaluate general health-related QoL and the European Organization for Research and Treatment of Cancer Quality of Life Questionnaire-breast cancer specific module (EORTC QLQ-BR23) to evaluate characteristic QoL of breast cancer patients.

The EQ-5D, a validated questionnaire translated into Korean, [11] is a widely-used questionnaire to evaluate general health status in 5 categories (mobility, self-care, usual activities, pain/discomfort, and anxiety/depression). Each category was assessed by 3 -grade Likert scale: 1 , No problem; 2, some or moderate problems; 3, extreme problems (EQ-5D-3 L). In addition, each category was also assessed on a visual analogue scale (VAS) where the endpoints are classified as follows: 'the best imaginable health state' $=100$ and the 'worst imaginable health state' $=0$ (EQ-5D-VAS).

The EORTC QLQ-BR23 instrument comprised of 23 questions, which can be divided into 8 categories and 2 areas which are functional (body image, future perspective, and sexual activities and enjoyment) and symptomatic (arm symptom, breast symptoms, side effects of systematic therapy, and upset by hair loss). Each question is scored on a 4-point Likert scale from 1 (not at all) to 4 (very much), and we arranged the data to the above-mentioned categorical basis and linearly transformed them into scores from 0 to 100; a higher score represented better satisfaction with their QoL. The Korean version of EORTC QLQ-BR23 has been tested in a previous study and was shown to be a valid measurement to assess QoL of breast cancer patients [12].

\section{Clinical and sociodemographic factors acquirement}

A researcher in each institution filled out the clinical factors, such as the time of surveillance, type of breast surgery, type of axillary surgery (axillary dissection, sentinel lymph node dissection, or none), chemotherapy, hormone therapy, target therapy, and the extent of radiotherapy. The sociodemographic factors and QoL were acquired by the self-administration method from patients. As for personal and sociodemographic factors, age, body mass index, menopausal status before diagnosis of breast cancer, periodic medication (cardiovascular, diabetic, and psychotropic drug), employment status, household income, education status, hobbies, alcohol consumption, smoking, and use of health supplement were considered.

In addition, the questionnaire of Global physical activity question (GPAQ) of the World Health Organization (WHO) to assess physical activeness was performed. The GPAQ was developed by the WHO for physical activity surveillance in many countries. It collects information on physical activity participation in activity at work, travel to and from places, and recreational activities. The questionnaire is comprised of questions asking about the number of days when a certain degree of activity is performed, and the actual time spent for the activity during the day, for last 7 days. The GPAQ was assorted into 3 grades according to total time of activity, which is calculated by multiplying the "number of days with certain activity per week' and 'actual time of activity in the day'.

The numerical values calculated through the above method were used for QoL analysis as a variable named physical activity. The GPAQ has been validated and applied in diverse studies worldwide including Asian countries $[13,14]$.

\section{Data assessment and statistical methods}

We assorted our study participants into 3 groups according to their surveillance period after the end of radiotherapy, which were the 1st year group (915 months), the 2nd year group (21-27 months), and the 3rd year group (33-39 months) from the end of radiotherapy. A chi-square test was performed to evaluate distribution of clinical and sociodemographic variables according to surveillance period.

For missing value control, we regarded clinical factors as missing value that chemotherapy less than 3 cycles, target therapy or hormone therapy less than 6 months. If each category of EQ-5D-VAS is only omitted with written EQ-5D-3 L, the omitted EQ-5D-VAS was replaced according to the median VAS for same grade of each category. In GPAQ, if our participants answered as 'I do not know/I am not sure' to the question asking actual time of activities, we replaced with median value of responders.

To identify which category of QoL is mostly deteriorated after breast cancer treatment, Cochran-MantelHaenszel analysis to assess the EQ-5D-3 L and multiple comparison test after Kruskal-Wallis analysis to assess EQ-5D-VAS and EORTC QLQ-BR23 were conducted.

For univariate analysis, Chi-square test or CochranMantel-Haenszel analysis was performed to investigate the relationship between various factors including the surveillance period, clinical and sociodemographic factors, and physical activity and EQ-5D-3 L.For EQ-5DVAS and EORTC QLQ-BR23, Kruskal-Wallis analysis was performed. Multiple regression analysis using stepwise methods were performed for the factors found to have a $p$ value $<0.1$ in univariate analysis for EQ-5DVAS and EORTC QLQ-BR23.All analyses were executed using the IBM SPSS statistics20 (IBM Inc., NY, USA) and the $\mathrm{R}$ version 3.2.0 (The $\mathrm{R}$ Foundation).

\section{Results}

\section{Characteristics of participants}

From August 2014 to September 2015, 1156 women from 17 hospitals consented and answered questionnaire. With regard to the surveillance period, 587 
(58.1\%), $332(30.7 \%)$ and $231(11.2 \%)$ patients were corresponded to the first, second and third year group, respectively. The sampling errors with 95\% confidence level of the first, second and third year groups were estimated as 3.8, 5.2 and $6.3 \%$, respectively. The surveillance period was missed in 6 questionnaires.

The clinical characteristics of study participants are shown in Table 1. Breast conserving surgery was performed in $91.9 \%$ of cases and patients with advanced stage of III or higher were in $11.4 \%$ of cases. Radiation field included the regional lymph nodes in $75 \%$ of cases and boost to tumor bed was performed in $89.7 \%$ of cases. Median dose to whole breast/chest wall and boost was 50.4 Gy and 10.0 Gy, respectively. Assorted by surveillance period, there was no statistically significant difference except for the variables of age and hormone therapy. The difference of age distribution may be reflected by the character of our study and as the surveillance period increases, the median age (50.0, 51.8 and 52.1 years at first, second and third year group, respectively) increases. Regarding hormone therapy, we have more study participants who did not received hormone therapy in the first year group than other groups (29.1 vs. 19.5 vs. 18.6) since some patients in first year group had received hormone therapy for less than 6 months and were considered as missing values. The sociodemographic status was assorted by the surveillance period and is represented in Table 2. There is no statistically significant difference in distribution except variables including hobbies. Regarding variables of hobbies, there are more participants who were categorized as 'actively participating' in the third year group.

\section{EQ-5D-3 L}

The answer rate of grade 2 or 3 was highest in the pain/ discomfort category, followed by anxiety/depression (Table 3). The grade 2 answer for the pain/discomfort category was significantly higher than expected. With a lapse of time, usual activity $(p=0.038)$ and pain/discomfort $(p<0.001)$ categories were improved, but the other categories showed no significant change. The trend that pain/discomfort grade 2 is measured as higher than expected, persisted in all surveillance periods. We summate grades of all 5 categories into total score ranged from 5 to 15 , to reflect general QoL. The total score showed significant improvement with lapse of time $(p=0.001)$.

\section{EQ-5D vas}

The EQ-5D VAS showed that pain/discomfort, anxiety/ depression, and mobility categories were significantly lower than usual activity and self-care categories. The same trend was observed in all surveillance periods. The categories of pain/discomfort $(p<0.001)$, self-care $(p=0.012)$ and usual activity $(p=0.022)$ were improved with a lapse of time (Fig. 1). We calculated the average scores of 5 categories to indicate general QoL, and the average score was improved with lapse of time $(p=0.001)$.

We performed multivariate analysis to factors showed to be significant in univariate analysis, and the clinical factors, such as stage of disease, surgery type, chemotherapy, hormone therapy, target therapy, and extent of radiotherapy did not affect EQ-5D-VAS significantly (Table 4). The axillary dissection (axillary dissection Vs. none) deteriorated the self-care, usual activities, pain/ discomfort, and the average score of EQ-5D-VAS $(p=0.039, p<0.001, p=0.004$, and $p=0.004$, respectively). With the lapse of time, the categories of pain/discomfort $(p<0.001)$, self-care $(p=0.034)$ and the average score $(p=0.004)$ were improved in multivariate analyses.

For sociodemographic factors, menopausal status $(p=0.003)$ before surgery, educational status $(p=0.014)$, household income $(p=0.001)$, employment change $(p=0.001)$, activity of hobbies $(p<0.001)$, physical activity $(p<0.001)$, and psychotropic medication $(p<0.001)$ were significant factors influencing the average score of EQ-5D. Psychotropic medication, employment change and activity of hobbies were found to be strongly related to anxiety/depression with $p<0.001$. Household income was a strong factor for pain/discomfort and mobility. Physical activity was another strong factor for mobility.

\section{EORTC QLQ-BR23}

The lowest scored category was sexual activity, followed by sexual enjoyment, future perspective, hair loss, body image, arm symptoms, side effects of systematic therapy, and breast symptoms. There were no significant differences between scores of body image, arm symptoms, and side effects of systematic therapy, while all the other categorical scores were significantly different between each other. These trends were observed in all surveillance periods. The categories of arm symptoms $(p<0.001)$ and breast symptoms $(p<0.001)$ were improved with time passage. Other categories showed no statistically significant change with time (Fig. 2.).

For upset by hair loss category, the answer rate was relatively low (55.6\%) as compared to other categories, since only the participants who experienced actual hair loss answered the question. There were some missing answers for sexual activity (6.1\%), and the answer rate of sexual enjoyment category was only $43.9 \%$, which is suggested to be answered from whom had had sexual activity.

In multivariate analysis, the axillary dissection (axillary dissection Vs. none) and stage of disease were significant 
Table 1 Clinical characteristics of survivors

\begin{tabular}{|c|c|c|c|c|c|}
\hline & & 1st year group & 2nd year group & 3 rd year group & \\
\hline & & $N(\%)$ & $N(\%)$ & $N(\%)$ & ${ }^{p}$ value ${ }^{\mathrm{a})}$ \\
\hline Age (years) & Median & 50.0 & 51.8 & 52.1 & 0.010 \\
\hline & $<41$ & $63(10.8)$ & $23(7.0)$ & $15(6.5)$ & \\
\hline & $41-50$ & $255(43.8)$ & $126(38.2)$ & $88(38.3)$ & \\
\hline & $51-60$ & $205(35.2)$ & $132(40.0)$ & $87(37.8)$ & \\
\hline & $>60$ & $59(10.1)$ & $49(14.8)$ & $40(17.4)$ & \\
\hline Stage of disease & & & & & 0.105 \\
\hline & 0 & $86(14.7)$ & $37(11.2)$ & $28(12.1)$ & \\
\hline & । & $250(42.8)$ & $156(47.4)$ & $107(46.3)$ & \\
\hline & $\|$ & $190(32.5)$ & $101(30.6)$ & $60(26.0)$ & \\
\hline & $\mathrm{III-IV}$ & $58(9.9)$ & $35(10.6)$ & $36(15.6)$ & \\
\hline Menstrual status & & & & & 0.383 \\
\hline & Premenopausal & $350(60.1)$ & $195(59.0)$ & $148(64.6)$ & \\
\hline & Postmenopausal & $232(39.9)$ & $135(40.9)$ & $81(35.4)$ & \\
\hline Breast surgery & & & & & 0.630 \\
\hline & Breast-conserving surgery & $541(92.6)$ & $302(91.2)$ & $210(90.9)$ & \\
\hline & Mastectomy & $43(7.4)$ & $29(8.8)$ & $21(9.1)$ & \\
\hline Axillary surgery & & & & & 0.206 \\
\hline & None & $62(10.7)$ & $27(8.2)$ & $25(10.9)$ & \\
\hline & Sentinel node dissection & $293(50.3)$ & $190(57.4)$ & $128(55.9)$ & \\
\hline & Axillary node dissection & $227(39.0)$ & $114(27.3)$ & $76(33.2)$ & \\
\hline Radiotherapy field & & & & & 0.905 \\
\hline & Breast only & $435(74.4)$ & $251(75.6)$ & $174(75.3)$ & \\
\hline & Breast and regional nodes & $150(25.6)$ & $81(24.4)$ & $57(24.7)$ & \\
\hline Chemotherapy & & & & & 0.469 \\
\hline & No & $211(36.3)$ & $125(37.8)$ & 75 (32.8) & \\
\hline & Yes & $371(63.7)$ & $206(62.2)$ & $154(67.2)$ & \\
\hline Hormone therapy & & & & & $<0.001$ \\
\hline & No & $163(29.1)$ & $64(19.5)$ & $43(18.6)$ & \\
\hline & Yes & $397(70.9)$ & $265(80.5)$ & $188(81.4)$ & \\
\hline Target therapy & & & & & 0.855 \\
\hline & No & 507 (88.9) & $294(89.4)$ & $203(87.9)$ & \\
\hline & Yes & $63(11.1)$ & 35 (10.6) & $28(12.1)$ & \\
\hline
\end{tabular}

a) Pearson's Chi-square test

clinical factors for the symptomatic area (Table 5). For the functional area, the type of breast surgery influenced body image, chemotherapy affected body image and sexual activity, and the axillary dissection (axillary dissection Vs. none) was the factor which affected sexual enjoyment. Various sociodemographic factors strongly affected each QoL category; household income for side effects of systematic therapy; activity of hobbies for side effects of systematic therapy, arm symptoms, future prospective and sexual activity; medication of psychotropic drug for side effects of systematic therapy and body image; employment change for body image and future prospective were observed with strong significance $(p<0.001)$.

\section{Discussion}

This study presented the general index of QoL with statistical power and showed the change in QoL in the critical early phase after adjuvant radiotherapy. We were able to observe the difference in QoL pattern with time during the first 3 years after breast cancer treatment. Our data of EQ-5D-3 L showed that pain/discomfort 
Table 2 Sociodemographic characteristics of survivors

\begin{tabular}{|c|c|c|c|c|c|}
\hline & \multirow{2}{*}{$\begin{array}{l}\text { 1st year group } \\
N(\%)\end{array}$} & \multirow{2}{*}{$\begin{array}{l}\text { 2nd year group } \\
N(\%)\end{array}$} & \multicolumn{2}{|l|}{ 3rd year group } \\
\hline & & & & $N(\%)$ & ${ }^{p}$ value $^{a)}$ \\
\hline Educational status & & & & & 0.238 \\
\hline & Low or middle & $350(61.0)$ & $193(59.6)$ & $152(66.4)$ & \\
\hline & High & $234(39.0)$ & $131(40.4)$ & 77 (33.6) & \\
\hline Any family memb & er living together & & & & 0.311 \\
\hline & Yes & $511(89.2)$ & $294(89.4)$ & $197(85.7)$ & \\
\hline & No & $62(10.8)$ & $35(10.6)$ & $33(14.3)$ & \\
\hline Change of employ & ment (before $\rightarrow$ after) & & & & 0.807 \\
\hline & Unemployed $\rightarrow$ Unemployed & $251(42.8)$ & $138(41.6)$ & $107(46.3)$ & \\
\hline & Employed $\rightarrow$ Unemployed & $123(21.0)$ & $69(20.8)$ & $42(18.2)$ & \\
\hline & Employed/Unemployed $\rightarrow$ Employed & $213(36.3)$ & $125(37.7)$ & $82(35.5)$ & \\
\hline Household incom & $(\$ / m o n t h)^{c}$ & & & & 0.594 \\
\hline & $<2500$ & $263(46.8)$ & $133(41.8)$ & $105(46.5)$ & \\
\hline & $2500-4000$ & $202(35.9)$ & $124(39.0)$ & $77(34.1)$ & \\
\hline & $>4000$ & $97(17.3)$ & $61(19.2)$ & $44(19.5)$ & \\
\hline Hobbies & & & & & 0.013 \\
\hline & Very active & $99(17.1)$ & $74(22.5)$ & $64(27.8)$ & \\
\hline & Moderate & $349(60.3)$ & $180(54.7)$ & $120(52.2)$ & \\
\hline & Rare & $131(22.6)$ & $75(22.8)$ & $46(20.0)$ & \\
\hline Smoking status (C & urrent) & & & & 0.116 \\
\hline & Non-smoker & $575(98.3)$ & $317(96.1)$ & $223(97.0)$ & \\
\hline & Smoker & $10(1.7)$ & $13(3.9)$ & $7(3.0)$ & \\
\hline Alcohol consumpt & & & & & 0.128 \\
\hline & No & 455 (77.6) & $265(80.3)$ & $168(73.0)$ & \\
\hline & Yes & $131(22.4)$ & $65(19.7)$ & $62(27.0)$ & \\
\hline Physical activity & & & & & 0.596 \\
\hline & High & $183(33.8)$ & $106(33.0)$ & $64(29.0)$ & \\
\hline & Moderate & $188(34.2)$ & $111(34.6)$ & $74(33.5)$ & \\
\hline & Low & $176(32.0)$ & $104(32.4)$ & $83(37.6)$ & \\
\hline
\end{tabular}

Pearson's Chi-square test

b) Low or Middle $=$ High school graduated or below, High $=$ College graduated or higher

${ }^{c}$ Household income is estimated to United State dollar from Korean won, with exchange rate of 1 USD for 1200 KRW. The cut-off values are one-third and two-third points according to Korean Statistics

Table 3 Result of the EQ-5D-3 L

\begin{tabular}{|c|c|c|c|c|c|c|c|c|c|c|c|c|}
\hline \multirow{3}{*}{ EQ-5D grade ${ }^{a)}$} & \multicolumn{3}{|l|}{ Total } & \multicolumn{3}{|c|}{ 1st year group } & \multicolumn{3}{|c|}{ 2nd year group } & \multicolumn{3}{|c|}{ 3rd year group } \\
\hline & 1 & 2 & 3 & 1 & 2 & 3 & 1 & 2 & 3 & 1 & 2 & 3 \\
\hline & $N(\%)$ & $N(\%)$ & $N(\%)$ & $N(\%)$ & $N(\%)$ & $N(\%)$ & $N(\%)$ & $N(\%)$ & $N(\%)$ & $N(\%)$ & $N(\%)$ & $N(\%)$ \\
\hline Mobility & 1009 (88.6) & $127(11.2)$ & $3(0.3)$ & $517(88.8)$ & $64(11.0)$ & $1(0.2)$ & $289(87.8)$ & 38 (11.6) & $2(0.6)$ & $203(89.0)$ & $25(11.0)$ & 0 \\
\hline Self-care & 1101 (96.7) & $33(2.9)$ & $4(0.4)$ & 562 (96.6) & $19(3.3)$ & $1(0.2)$ & 318 (97.0) & $7(2.1)$ & $3(0.9)$ & 221 (96.9) & $7(3.1)$ & 0 \\
\hline Usual activities & $984(86.4)$ & $153(13.4)$ & $2(0.2)$ & $492(84.5)$ & 89 (15.3) & $1(0.2)$ & $286(86.9)$ & $43(13.1)$ & 0 & $206(90.4)$ & $21(9.2)$ & $1(0.4)$ \\
\hline Pain/Discomfort & $552(48.5)$ & $574(50.4)$ & $12(1.1)$ & $248(42.6)$ & $328(56.4)$ & $6(1.0)$ & 166 (50.6) & 159 (48.5) & $3(0.9)$ & $138(60.5)$ & 87 (38.2) & $3(1.3)$ \\
\hline $\begin{array}{l}\text { Anxiety/ } \\
\text { Depression }\end{array}$ & 655 (57.6) & $470(41.3)$ & $13(1.1)$ & $324(55.7)$ & 254 (43.6) & $4(0.7)$ & 189 (57.6) & $135(41.2)$ & $4(1.2)$ & $142(62.3)$ & 81 (35.5) & $5(2.2)$ \\
\hline$p$ value & & & $<0.001$ & & & $<0.001$ & & & $<0.001$ & & & $<0.001$ \\
\hline
\end{tabular}

Abbreviation: EQ-5D 3 L EuroQol group 5-dimensional 3-Likert scale questionairre

${ }^{\text {a) }}$ Grade 1 = No problem, Grade 2 = Some/moderate problem, Grade 3 = Extreme problem

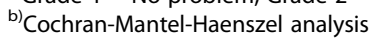



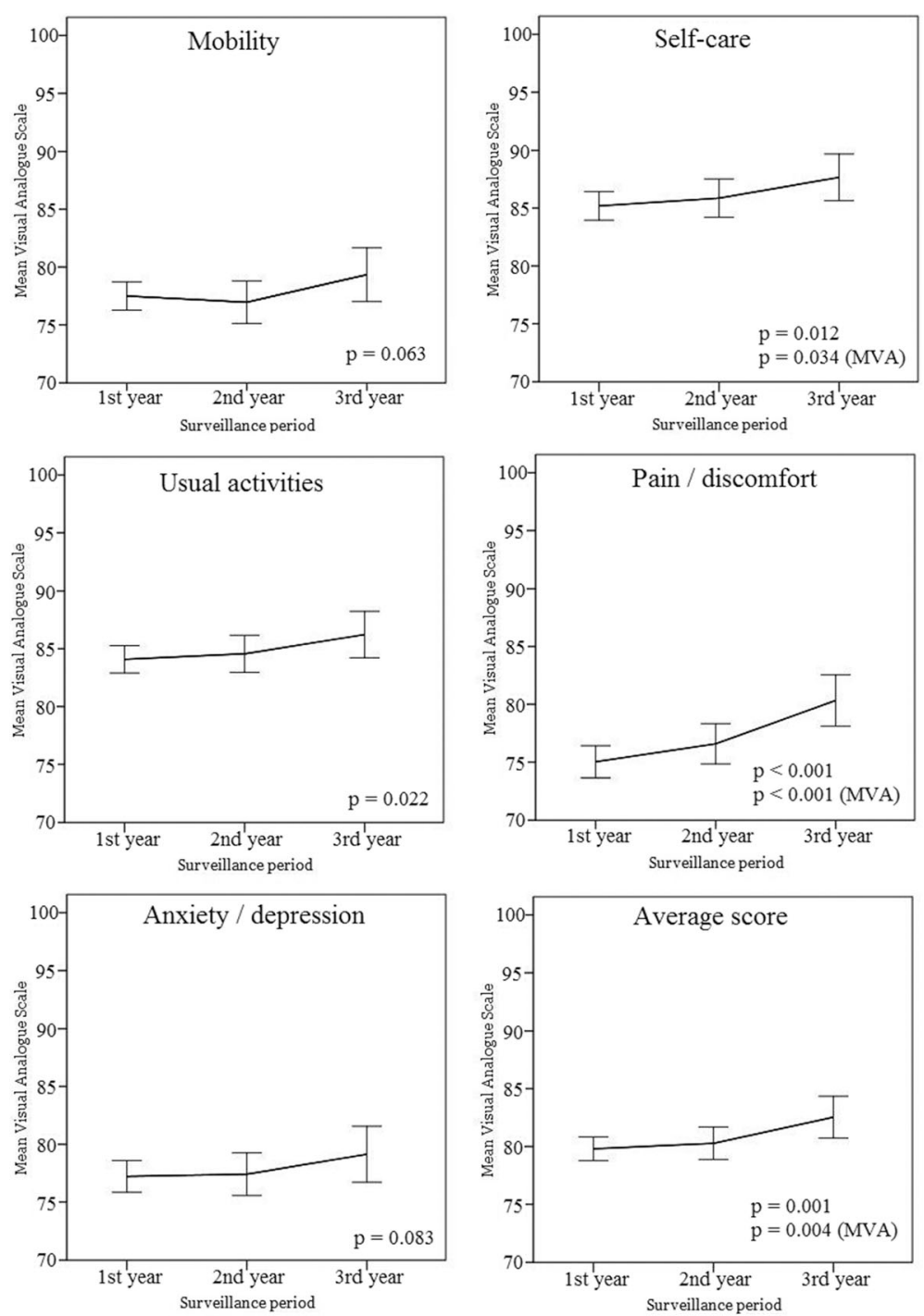

Fig. 1 Longitudinal comparison of EQ-5D VAS scores according to surveillance period. Higher scores represented better satisfaction with their quality of life. The $95 \%$ confidence intervals are represented th the each point of surveillance period. Statistically significant $p$-values through multivariate analysis (comparing 1st year and 3rd year) are shown. Abbreviations: MVA, multivariate analysis

and anxiety/depression were the most deteriorated categories. Regarding EORTC QLQ-BR23, hair loss in the symptom area and sexual activity in the function area were the most unsatisfactory categories in each area, for all surveillance periods.

Although there is little previous data investigating the priority between categories of EQ-5D or EORTC QLQBR23, our study showed similar results with other studies. Kim et al. performed a study with breast cancer patients after treatment, measuring QoL with instruments including EQ-5D. Between 5 categories of EQ-5D, far more patients answered with higher grades in categories of pain/discomfort and anxiety/depression [11]. In other studies that used EORTC QLQ-BR23 with breast cancer patients who completed treatment including radiotherapy, the upset by hair loss category had the lowest score in the symptom area [4]. Referring to another Korean study assessing QoL of breast cancer, sexual activity was the far lowest scored category in the functional area [15].

Several studies have reported that breast cancer survivors showed improvement over time in many domains of QoL including pain, breast and arm symptoms $[16,17]$. The authors of a 10 year long-term follow up study reported that the symptoms of pain improved 3 years after treatment but aggravated 5 to 10 years after treatment [8]. In a recent study with early breast cancer patients who completed treatment including radiotherapy, the breast symptoms category of EORTC QLQ-BR23 was improved at 6-8 months and 2 years after treatment [18]. 


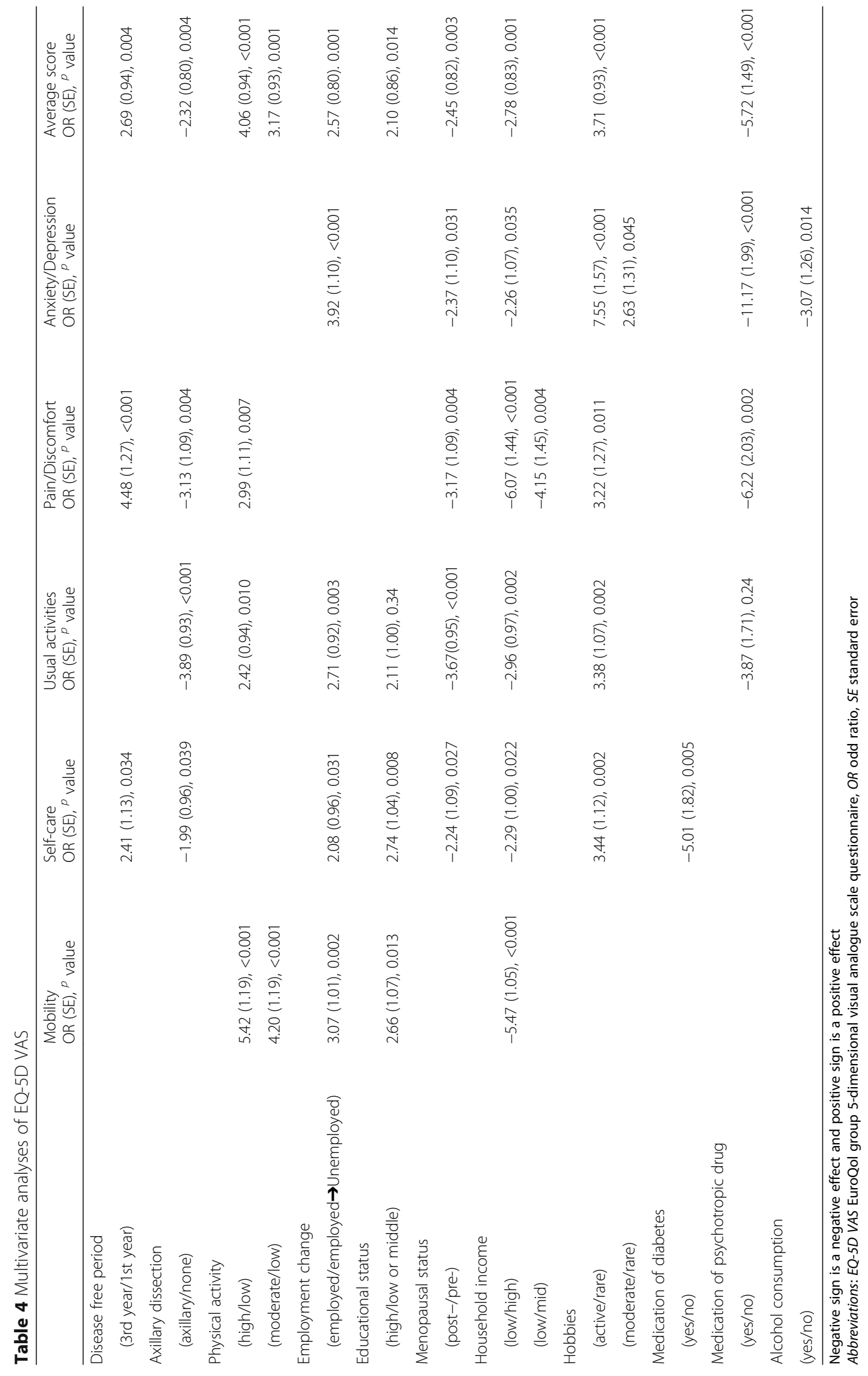



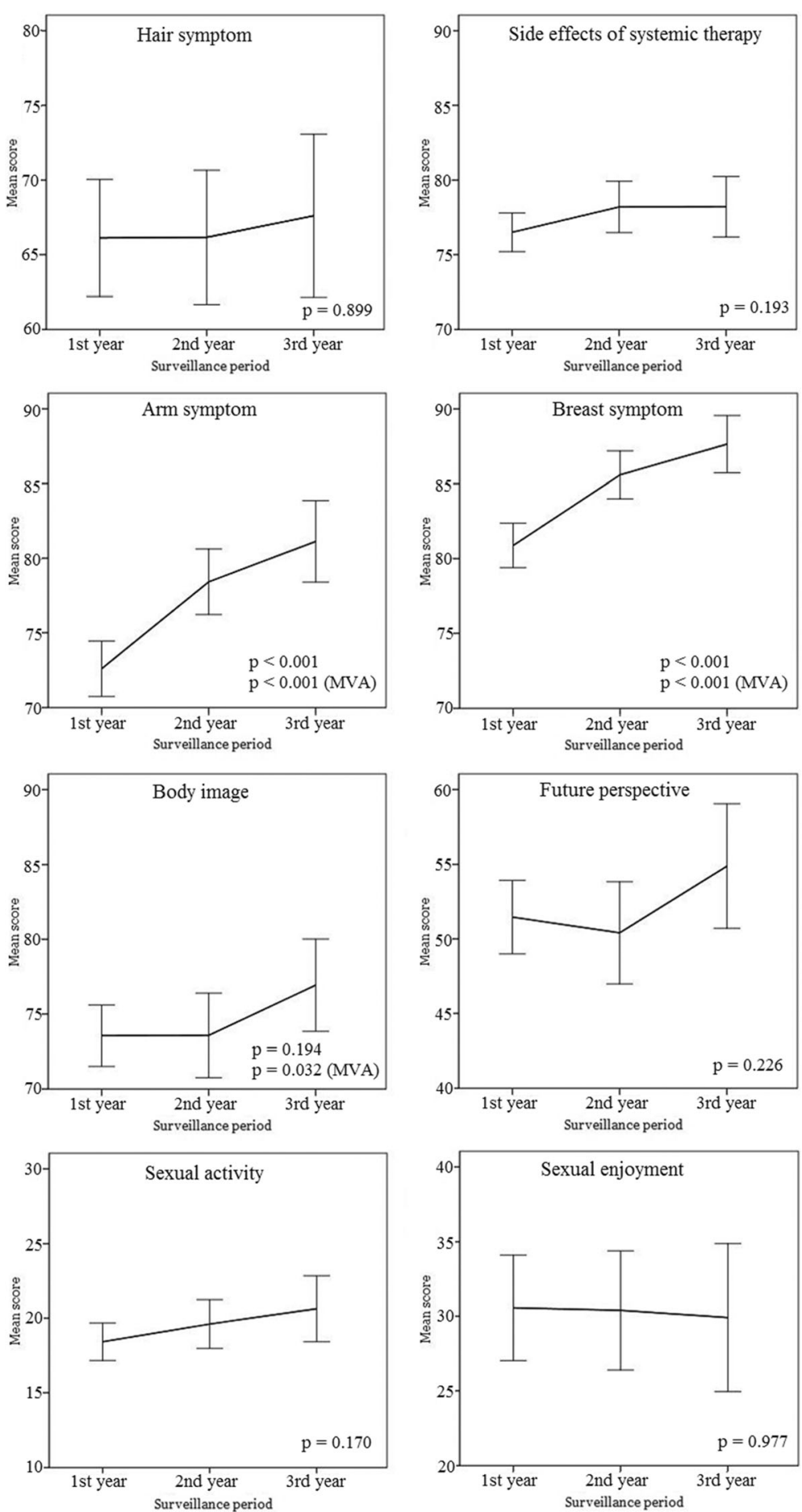

Fig. 2 Longitudinal comparison of EORTC BR-23 scores according to surveillance period. Higher scores indicate better health-related quality of life. The $95 \%$ confidence intervals are marked at the each point of surveillance period. Statistically significant $p$-values through multivariate analysis (comparing 1st year and 3rd year) are shown. Abbreviations: MVA, multivariate analysis

A French prospective study using EORTC QLQ-BR23 also showed that breast symptoms and arm symptom categories were significantly improved 3 and 6 months after surgery [19]. Our present study showed corroborated results that breast symptoms and arm symptom categories of EORTC BR-23 were significantly improved with the lapse of time. Improvement in EQ-5D VAS categorical data of pain/discomfort for the first 


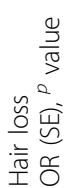

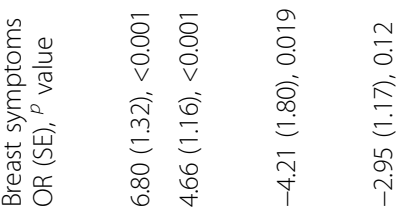

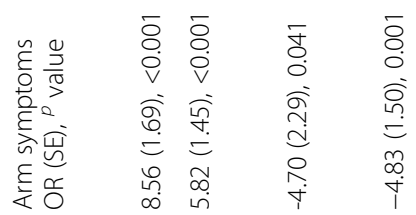

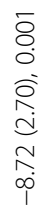

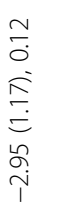

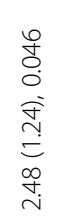

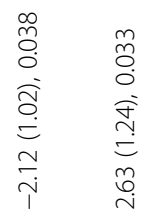

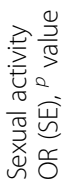

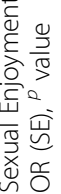

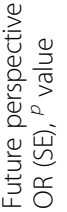

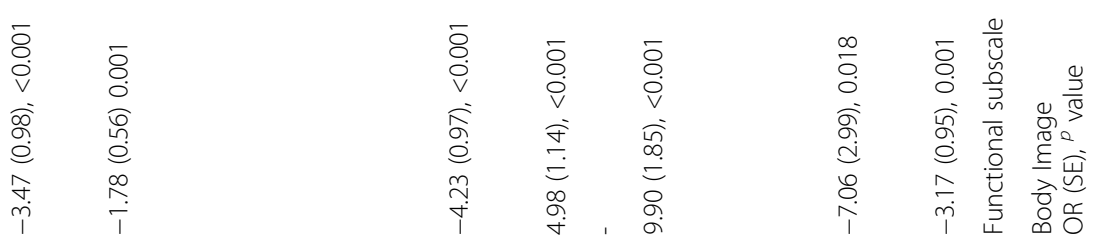

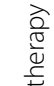

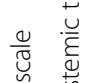

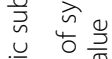

证

密出

究

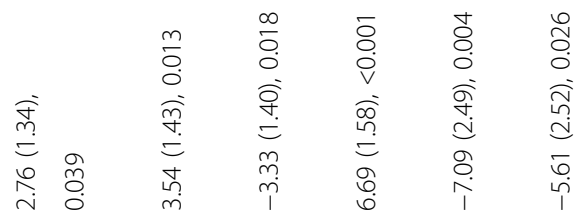
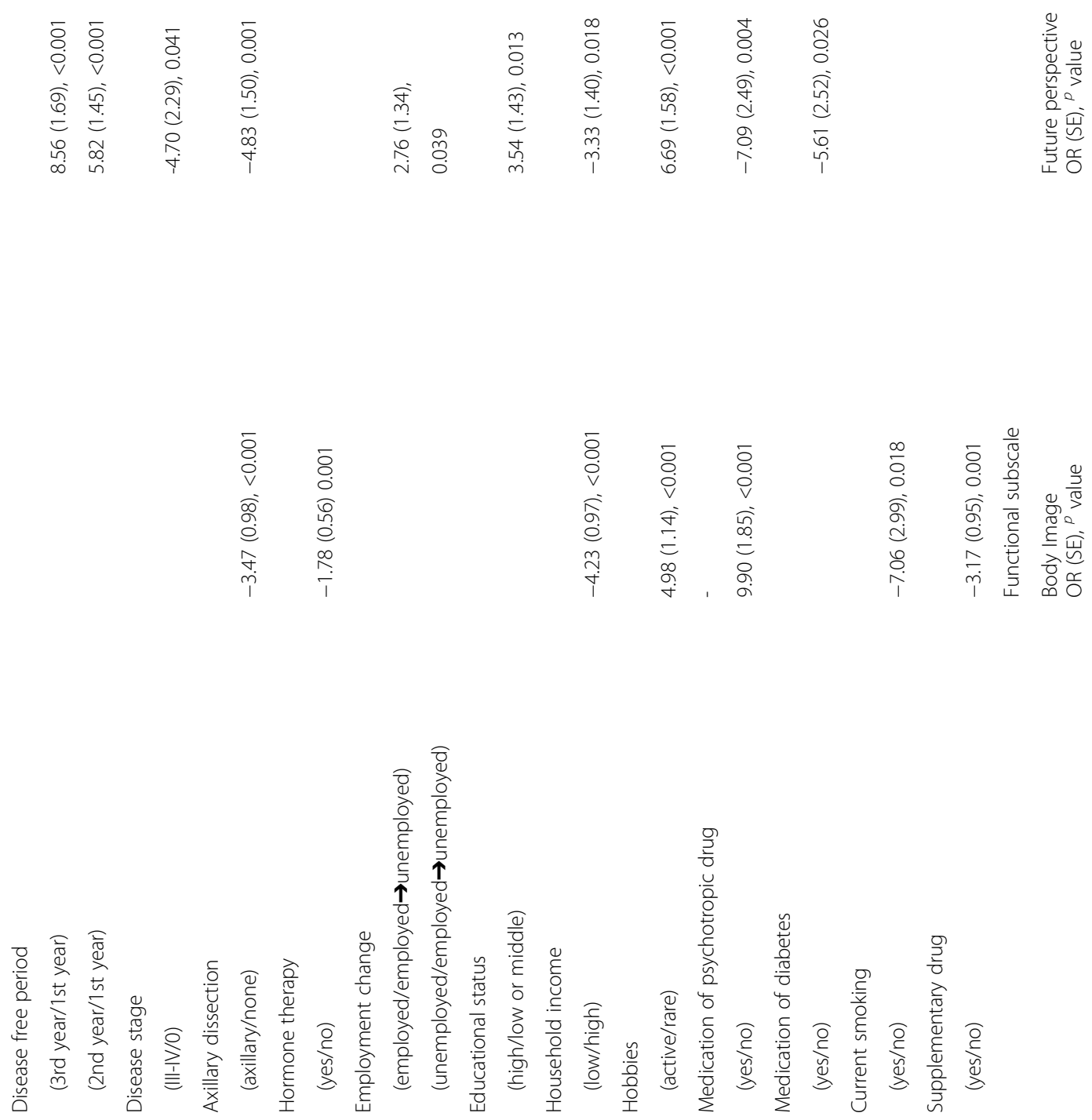


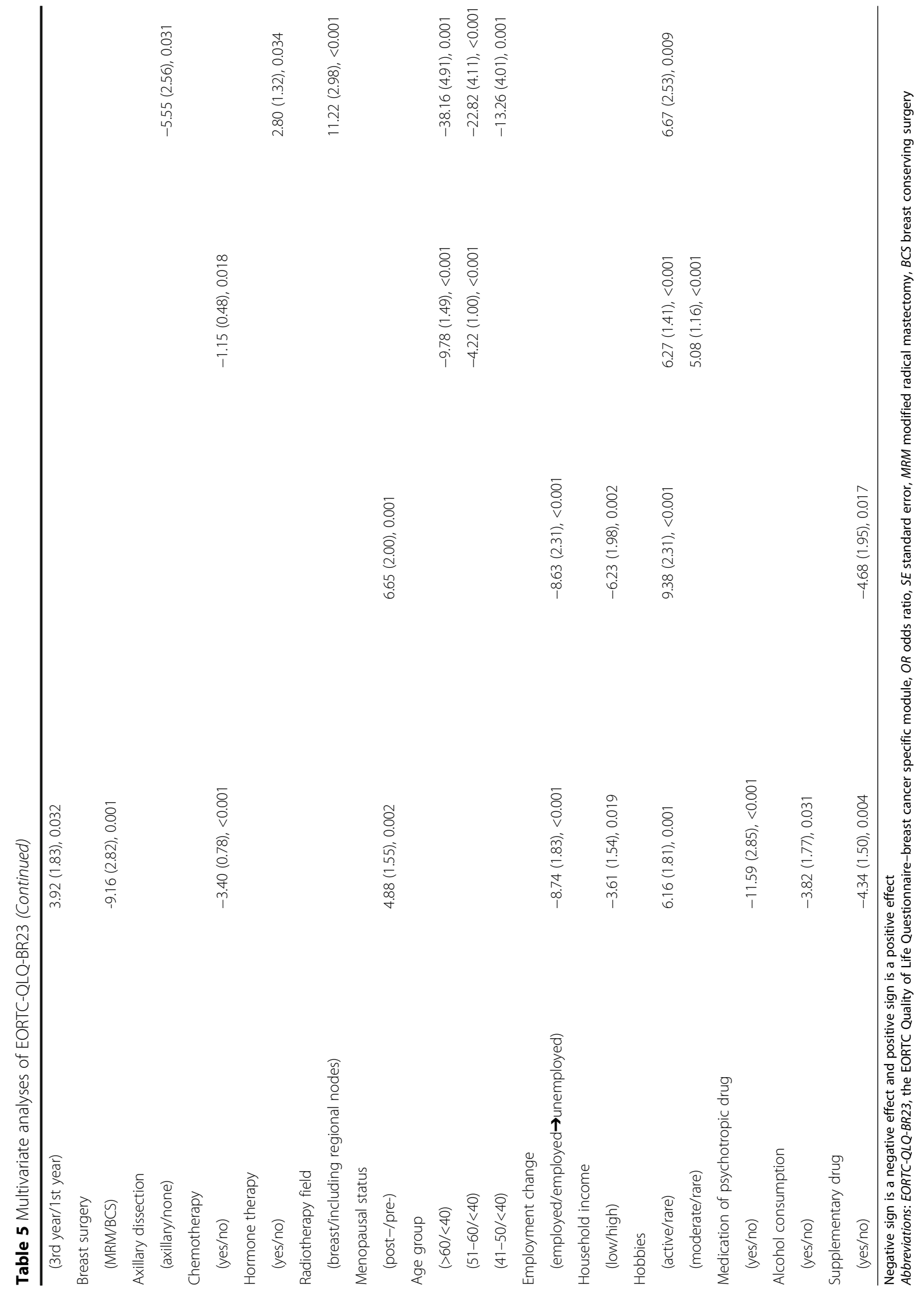


3 years after treatment is similar with the above mentioned study $[8,16,17]$.

In out study, anxiety/depression category in EQ-5D VAS and future perspective category in EORTC QLQBR23, which reflect mental aspect, were not significantly improved. Psychosocial support may be needed considering their mental burden. Another point is that the categories of sexual activity and enjoyment in EORTC QLQ-BR23 scored far lower than other categories. We assume that the result is owing to atmosphere affected by Confucianism culture, where it is uncommon and difficult to discuss the sexual subject.

Various clinical and sociodemographic factors were investigated in our study. Multivariate analysis revealed that the axillary dissection was a prominent clinical factor affecting the largest number of QoL categories. Axillary dissection is the factor that might cause lymphedema related arm symptoms or other functional side effect, [20] hence postoperative rehabilitation is recommended and an effort to spare aggressive dissection should be continued. Many sociodemographic factors affected several categories of QoL in multivariate analysis; household income, activeness of hobbies, educational status, and occupational status were prominent. Other investigators also mentioned that socioeconomic factors were important determinants of QoL, including educational status, household income, and occupational factors [21, 22]. Some sociodemographic factors are difficult to change after cancer treatment; however, physical activity and activeness of hobbies could be boosted by various social programs. Previous studies using exercise intervention reported the positive impact on QoL such as depression, body image and sleep quality $[23,24]$.

The limitation of this study is that the data at each year was not derived from same patients, so we can only compare the data of population in relevant periods. Despite this disadvantage, this study is meaningful with investigation for a large number of patients in a multicenter group, and comprehensive categorical analysis of time-related QoL changes.

\section{Conclusion}

Pain/discomfort and self-care categories in EQ-5D VAS, arm/breast symptoms and body image in QLQ-BR23 were improved during follow-up. On the other hand, Anxiety/depression in EQ-5D VAS and future perspective QLQ-BR23 has not improved, suggesting necessity of psychosocial support. Axillary dissection affected many categories of QoL and should be spared if possible, and various sociodemographic factors affect QoL, not only clinical factors. These results may be useful for follow-up consultation after treatment of breast cancer patients.

\section{Abbreviations}

3 L: 3-Grade likert scale; EQ-5D: The 5-dimensional questionnaire by the EuroQo group; GPAQ: Global physical activity question; MVA: Multivariate analysis; QLQBR23: The EORTC Quality of Life Questionnaire-breast cancer specific module; QoL: Quality of life; VAS: Visual-analogue scale; WHO: World Health Organization

\section{Acknowledgement}

Not applicable.

\section{Funding}

None to be declared.

\section{Availability of data and materials}

The datasets used and/or analysed during the current study available from the corresponding author on reasonable request.

\section{Authors' contributions}

CHR was a major contributor in writing the manuscript, and WSY designed study and analyzed the patient data regarding the information of questionaiire and clinical data. All authors read and approved the final manuscript.

\section{Competing interests}

The authors declare that they have no competing interests.

\section{Consent for publication}

Not applicable.

\section{Ethics approval and consent to participate}

After the review and approval of study protocol from Korean Radiation Oncology Group, ethical approval was obtained from each hospital Institutional Review Board. All procedures performed in studies involving human participants were in accordance with the ethical standards of the institutional and/or national research committee and with the 1964 Helsinki declaration and its later amendments or comparable ethical standards.

Informed consent was obtained from all individual participants included in the study.

\section{Author details}

${ }^{1}$ Department of Radiation Oncology, Yonsei Cancer Center, Yonsei University College of Medicine, Seoul, Republic of Korea. ${ }^{2}$ Department of Radiation Oncology, Chonnam National Univiersity Hwasun Hospital, Hwasun, Jeollanam-do, Republic of Korea. ${ }^{3}$ Department of Radiation Oncology, Dongsan Medical Center, Keimyung University, Daegu, Republic of Korea. ${ }^{4}$ Department of Radiation Oncology, Ansan Hospital, Korea University, 123 Jeokgeum-ro, Danwon-gu, Ansan, Gyeonggi-do 15355, Republic of Korea. ${ }^{5}$ Department of Radiation Oncology, Ajou University, Suwon, Gyeonggi-do, Republic of Korea. ${ }^{6}$ Department of Radiation Oncology, Guro Hospital, Korea University, Seoul, Republic of Korea. ${ }^{7}$ Department of Radiation Oncology, St. Vincent's Hospital, College of Medicine, The Catholic University of Korea, Seoul, Republic of Korea. ${ }^{8}$ Department of Radiation Oncology, Seoul National University Hospital, Seoul, Republic of Korea. ${ }^{9}$ Department of Radiation Oncology, Mokdong Hospital, Ewha Woman's University School of Medicine, Seoul, Republic of Korea. ${ }^{10}$ Department of Radiation Oncology, Kyung Hee University Medical Center, Kyung Hee University School of Medicine, Seoul, Republic of Korea. ${ }^{11}$ Department of Radiation Oncology, Boramae Medical Center, Seoul National University, Seoul, Republic of Korea. ${ }^{12}$ Department of Radiation Oncology, Cheil General Hospital and Women's Healthcare Center, Dankook University College of Medicine, Seoul, Republic of Korea.

${ }^{13}$ Department of Radiation Oncology, Sanggye Paik Hospital, Inje University, Seoul, Republic of Korea. ${ }^{14}$ Department of Radiation Oncology, Kosin University Gospel Hospital, Busan, Republic of Korea. ${ }^{15}$ Radiation Oncology, Gyeongsang National University School of Medicine and Gyeongsang National Univeristy Hospital, Jinju, Gyeongsangnam-do, Republic of Korea.

${ }^{16}$ Department of Radiation Oncology, Asan Medical Center, University of Ulsan College of Medicine, Seoul, Republic of Korea. ${ }^{17}$ Department of Radiation Oncology, Uijeongbu St. Mary's Hospital, The Catholic University of Korea, Uijeongbu, Gyeonggi-do, Republic of Korea. ${ }^{18}$ Department of Medical Science Research Center, Ansan Hospital, Korea University, Ansan,

Gyeonggi-do, Republic of Korea. 
Received: 24 January 2017 Accepted: 2 May 2017

Published online: 10 May 2017

\section{References}

1. Torre LA, Bray F, Siegel RL, Ferlay J, Lortet-Tieulent J, Jemal A. Global cancer. Statistics, 2012. CA Cancer J Clin. 2015;65:87-108.

2. Youlden DR, Cramb SM, Dunn NA, Muller JM, Pyke CM, Baade PD. The descriptive epidemiology of female breast cancer: an international comparison of screening, incidence, survival and mortality. Cancer Epidemiol. 2012;36:237-48.

3. Cancer Statistics. Available via National Cancer Information Center, Ministry of Health and Welfare. http://www.cancer.go.kr. Accessed 29 Jan 2016.

4. Munshi A, Dutta D, Kakkar S, Budrukkar A, Jalali R, Sarin R, Gupta S, Parmar V, Badwe R. Comparison of early quality of life in patients treated with radiotherapy following mastectomy or breast conservation therapy: a prospective study. Radiother Oncol. 2010;97:288-93.

5. Early Breast Cancer Trialists' Collaborative G, Darby S, McGale P, Correa C, Taylor C, Arriagada R, Clarke M, Cutter D, Davies C, Ewertz M, et al. Effect of radiotherapy after breast-conserving surgery on 10-year recurrence and 15 year breast cancer death: meta-analysis of individual patient data for 10,801 women in 17 randomised trials. Lancet. 2011;378:1707-16.

6. Ebctcg MGP, Taylor C, Correa C, Cutter D, Duane F, Ewertz M, Gray R, Mannu G, Peto R, et al. Effect of radiotherapy after mastectomy and axillary surgery on 10-year recurrence and 20-year breast cancer mortality: metaanalysis of individual patient data for 8135 women in 22 randomised trials. Lancet. 2014:383:2127-35.

7. Bloom JR, Petersen DM, Kang SH. Multi-dimensional quality of life among long-term (5+ years) adult cancer survivors. Psychooncology. 2007;16:691-706.

8. Koch L, Jansen L, Herrmann A, Stegmaier C, Holleczek B, Singer S, Brenner $\mathrm{H}$, Arndt V. Quality of life in long-term breast cancer survivors - a 10-year longitudinal population-based study. Acta Oncol. 2013;52:1119-28.

9. Jung KW, Park S, Won YJ, Kong HJ, Lee JY, Park EC, Lee JS. Prediction of cancer incidence and mortality in Korea, 2011. Cancer Res Treat. 2011;43:12-8.

10. Siegel R, DeSantis C, Virgo K, Stein K, Mariotto A, Smith T, Cooper D, Gansler T, Lerro C, Fedewa $S$, et al. Cancer treatment and survivorship statistics, 2012. CA Cancer J Clin. 2012;62:220-41.

11. Kim SH, Jo MW, Lee JW, Lee HJ, Kim JK. Validity and reliability of EQ-5D-3L for breast cancer patients in Korea. Health Qual Life Outcomes. 2015;13:203.

12. Yun YH, Bae SH, Kang IO, Shin KH, Lee R, Kwon SI, Park YS, Lee ES. Crosscultural application of the Korean version of the European Organization for Research and Treatment of cancer (EORTC) breast-cancer-specific quality of life questionnaire (EORTC QLQ-BR23). Support Care Cancer. 2004;12:441-5.

13. Au TB, Blizzard L, Schmidt M, Pham LH, Magnussen C, Dwyer T. Reliability and validity of the global physical activity questionnaire in Vietnam. J Phys Act Health. 2010;7:410-8.

14. Bull FC, Maslin TS, Armstrong T. Global physical activity questionnaire (GPAQ): nine country reliability and validity study. J Phys Act Health. 2009;6:790-804

15. Song S, Hwang E, Moon HG, Noh DY, Lee JE. Adherence to guidelines for cancer survivors and health-related quality of life among Korean breast cancer survivors. Nutrients. 2015;7:10307-19.

16. Ganz PA, Kwan L, Stanton AL, Bower JE, Belin TR. Physical and psychosocial recovery in the year after primary treatment of breast cancer. J Clin Oncol. 2011:29:1101-9.

17. Hsu T, Ennis M, Hood N, Graham M, Goodwin PJ. Quality of life in long-term breast cancer survivors. J Clin Oncol. 2013:31:3540-8.

18. Garsa AA, Ferraro DJ, DeWees TA, Deshields TL, Margenthaler JA, Cyr AE, Naughton M, Aft R, Gillanders WE, Eberlein T, et al. A prospective longitudinal clinical trial evaluating quality of life after breast-conserving surgery and high-dose-rate interstitial brachytherapy for early-stage breast cancer. Int J Radiat Oncol Biol Phys. 2013;87:1043-50.

19. Anota A, Bascoul-Mollevi C, Conroy T, Guillemin F, Velten M, Jolly D, Mercier M, Causeret S, Cuisenier J, Graesslin O, et al. Item response theory and factor analysis as a mean to characterize occurrence of response shift in a longitudinal quality of life study in breast cancer patients. Health Qual Life Outcomes. 2014;12:32

20. Gartner R, Jensen MB, Kronborg L, Ewertz M, Kehlet H, Kroman N. Selfreported arm-lymphedema and functional impairment after breast cancer treatment-a nationwide study of prevalence and associated factors. Breast. 2010;19:506-15.
21. Ashing-Giwa KT, Lim JW. Examining the impact of socioeconomic status and socioecologic stress on physical and mental health quality of life among breast cancer survivors. Oncol Nurs Forum. 2009;36:79-88.

22. Chang O, Choi EK, Kim IR, Nam SJ, Lee JE, Lee SK, Im YH, Park YH, Cho J. Association between socioeconomic status and altered appearance distress, body image, and quality of life among breast cancer patients. Asian Pac J Cancer Prev. 2014;15:8607-12.

23. Mishra SI, Scherer RW, Geigle PM, Berlanstein DR, Topaloglu O, Gotay CC, Snyder C. Exercise interventions on health-related quality of life for cancer survivors. Cochrane Database Syst Rev. 2012;8:CD007566.

24. Mustian KM, Sprod LK, Janelsins M, Peppone LJ, Palesh OG, Chandwani K, Reddy PS, Melnik MK, Heckler C, Morrow GR. Multicenter, randomized controlled trial of yoga for sleep quality among cancer survivors. J Clin Oncol. 2013;31:3233-41.

\section{Submit your next manuscript to BioMed Central and we will help you at every step:}

- We accept pre-submission inquiries

- Our selector tool helps you to find the most relevant journal

- We provide round the clock customer support

- Convenient online submission

- Thorough peer review

- Inclusion in PubMed and all major indexing services

- Maximum visibility for your research

Submit your manuscript at www.biomedcentral.com/submit

) Biomed Central 